\title{
A Structured-Population Genetic-Algorithm based on Hierarchical Hypercube of Genes Expressions
}

\author{
Mohamed A. Belal \\ Computer Science Department \\ Helwan University, Egypt
}

\author{
Mohamed H. Haggag \\ Computer Science Department \\ Helwan University, Egypt
}

\begin{abstract}
Structured-population Genetic Algorithm (GA) usually leads to more superior performance than the panmictic genetic algorithm; since it can control two opposite processes, namely exploration and exploitation in the search space. Several spatially structured-population GAs have been introduced in the literature such as cellular, patchwork, island-model, terrain-based A, graph-based, religion-based and social-based GA. All the aforementioned works did not construct the subpopulations based on the genes information of the individuals themselves. The structuring of sub-populations based on this information might help in attaining better performance and more efficient search strategy. In this paper, the structured population is represented as hierarchical hypercube of subpopulations that are dynamically constructed and adapted at search time. Each sub-population represents a sub-division of the real genes space. This structure could help in directing the search towards the promising sub-spaces. Finally, a comparative study with other known structured population $\mathrm{GA}$ is provided.
\end{abstract}

\section{Keywords}

Evolutionary Algorithms, Genetic Algorithms, Structured Population, Gene Expression

\section{INTRODUCTION}

The standard or panmictic model of GA, where the population is not explicitly structured, is not sufficiently powerful to solve complex problems. Individuals can interact genetically with each other. Traditionally, a hybrid approach of the standard GA and other technologies is used, such as the works in [1,2 and 3] in order to provide a reliable solution to the real-world problems.

The standard GA with its unstructured population encounters two problems while being applied to real-world problems; namely slow convergence and premature convergence. The slow convergence is attributed to the randomness in all the standard GA operations. On the other hand, premature convergence occurs due to the uneven distribution of the population over the search space. As a result, there is a problem of exploration/exploitation tradeoff, in other words more exploration leads to random walk on search space, and more exploitation could result in trapping at local optima.

For the aforementioned reasons, several structured-population GAs were introduced. In contrast with unstructured GA, the populations are divided into sub-populations; this division restricts the recombination operations in structured-population GA. Structured population results in better performance than panmictic GA, as it provides faster convergence and better population diversity, [4 and5].

Interactions among individuals in the population pool of structured-population GAs support better exploration and exploitation of the search space. Structured-population GAs can be classified as spatial segregation, spatial distance and heterogeneous population. The reproduction process is controlled by some factors like aging, social behavior and restricting randomness [42].

Examples of structured-population based GAs include cellular GA [6], island GA [7], patchwork GA [8 and 9], terrain-based GA [10] and religion-based GA [11]. Usually, structuredpopulation GA approaches are more common in implementations when GA is executed on parallel computers [12]. Another important and related subject is the issue of self-adapting parameters of GA, which has the potential of adjusting the algorithm while solving the required problem [8].

Evolutionary Algorithms (EA) generate solutions to optimization problems using techniques inspired by natural evolution. Evolution in stage structured populations was previously available only for populations structured by age. Consequently, evolution prediction and demography of stagestructured populations was introduced by [41]. Age- versus stage-structured populations evolution rates showed biological insights about evolution in stage-structured populations.

All the preceding approaches do not relate the spatially structured populations to the space of possible solutions, which are encoded in the genes of the individuals' chromosomes as presented in this paper. In my opinion, this relation is important as it helps in distributing the individuals over all the search space. It also enables the search algorithm to discover portions of the search spaces that are more likely to have optima. Moreover, it could preserve the diversity by keeping a minimum number of individuals at each search subspace. Finally, it supports local search by restricting recombination in the same subspace or the immediate neighborhood.

In the next section, a survey on the related works is provided. In section 3 , the proposed model and algorithm as well as the GA operations of the model are introduced. In section 4, the simulation and the results are presented followed by the conclusion.

\section{RELATED WORKS}

The first spatially structured population GA introduced was the Island-based Genetic Algorithm (IGA) [13]. IGA is a distributed version of genetic algorithms in which independent populations (islands) are evolving separately with the ability of exchanging chromosomes between each other. These remote chromosomes can prevent premature convergence in a population. Another advantage of IGA is that each island can use different GA operators, which causes different convergence rates. Island model can solve more complex problems that the standard model failed to [14 and 15]. IGA is considered coarse-grained parallel GA in which individuals migrate between islands according to a defined 
topology [16]. Various topologies have been proposed, such as ring, tree and hypercube topologies [17, 18 and 19].

The second spatially structured population GA was Cellular GA [6] (CGA). The structure of the population in this approach is the grid, where each cell in the grid represents one individual and it interacts only with its neighborhood. This representation creates some kind of local structure. Additionally, it enhances the population diversity and the exploration by causing slow diffusion of solutions. The term cellular genetic algorithm was proposed, in the literature to describe the mapping of GA to a parallel architecture $[6,20$, 21 and 22].

Patchwork model [7] is a mix between the island model and the cellular model of genetic algorithms. In Patchwork model, the population size can vary and the model leads to better control of both the population diversity and the selective pressure. Its operations are local in scope, and it adds 'behavioral rules' for individuals.

A model for the evolution of spatially structured populations of asexual haploid organisms was considered by [44] based on adaptation process. A local competition for replication is introduced, where each organism interacts only with its nearest neighbors. Evaluation ascertains that the stochastic processes governing the populations' evolution in the structured model are more effective than in the unstructured case. Additionally, the substitution rate of beneficial mutations is smaller for a spatially structured population than that seen for populations without structure. Increasing the adaptive mutation increases the diff erence between structured and unstructured populations. As the number of neighbors for the local competition is reduced, the substitution rate decreases as.

The terrain-based model is a self-tuning version of cellular GA in which the dimensions of the grid are interpreted as the mutation rate and the number of crossover points [10]. Other works put constraints on mating based on religion, such as religion-based model [10], or based on actual social relations such as social-based GA [23].

Integration of hypercube model, a location model and genetic algorithm was provided by [39] to obtain appropriate unit locations in a two-step approach. The first step is concerned with service area partitioning into superdistricts and determining the necessary number of units for each superdistrict. An approximate resolution for symmetric hypercube model with spatially homogeneous demand is provided. Then, a hypercube GA obtains best superdistricts and associates unit numbers. The second step provides optimal deployment of units within each superdistrict.

In general, spatially structured population could be represented by any graph of sub-populations that could control the evolution procedure. For instance, a graph-based GA was used to structure the population in [24]. Another example is the hierarchically structured population. This hierarchically structured population is used for flowshop scheduling in [12], where the number of possible neighborhoods is limited by dividing the population into clusters.

Six decades of catch-at-age data for perch and pike [40] in Windermere (UK) was used to estimate age- and sex-specific population sizes, natural mortalities and catch abilities in both species. Population sizes are estimated using standard maximum likelihood methods. Approach has been validated through estimates addressing important aspects of fisheries biology. It has been indicated that strong fishery selection against male perch apparently triggered a population collapse.

Population size is a critical parameter in GA in which the quality of the solution depends on [25]. Several approaches that automatically adjust GA's parameters including population size are introduced [26 and 27]. This problem is difficult since it depends on the problem required to be solved. Other approaches introduced what is called meta-GA to adjust these parameters [28]. In earlier studies, a subpopulations competition is used as a strategy to adapt the population size of each sub-population while the whole population size is fixed [29]. Three factors considered by [43] to describe the influence on population size. They are the natural growth of sub-populations, the internal competition of individuals from same sub-population and the interaction between individuals from different sub-populations.

The diversity of population has a great role in the quality of the output [30 and 31]; as it avoids the premature convergence [32]. Even the diversity of the initial population affects the performance of the GA [30 and 31]. The proposed model controls the diversity of the population by keeping a minimum number of individuals at each sub-space.

Some models, such as the patchwork model [3], strategy for Coevolutionary Algorithm (CEA) [43] and others, try to adapt the size of populations. Much work has been done to selfadaptively adjust the population size during an evolutionary process, with most focuses on how to vary the population size for GA [43]. Population sizing is should be set proportionally to the problem's size and difficulty. For real world problems, problem difficulty is very hard to estimate and accordingly the difficulty of setting the appropriate population size, which is a motivation for adjusting the population schemes. Other motivations are to get a better solution quality and better performance than a GA with a fixed population size and to make life easier for users by eliminating the population sizing parameter [45].

The size of the population, whether fixed or varied, plays a significant role in the performance of GA as well [33 and 34]. Both small population [35] and large [36] population are not required in GAs. Consequently, it remains an open problem [34]. Some authors applied self-adaptation of the population size in order to tackle this problem [37 and 38].

\section{THE PROPOSED MODEL}

Unlike the fixed topology of the structured population in both island and diffusion models, the introduced model in this paper has an adaptive topology structure which implicitly control the search directions and the concentration of individuals over the real search space.

The proposed model has the distributed nature of IGA. However, there is no migration in this model. Conversely, it has local interactions similar to the diffusion characteristic of CGA. Moreover, it has the self-adaptation nature of Patchwork model since the number of individuals and the search direction is determined autonomously.

The introduced model is based on local interaction between the actual problem's subspaces. This distributed structure permits the global behavior to evolve naturally out of all the local interactions. The reproduction process depends completely on local genes information in the gene subspaces. In other words, genetic operators are local in scope. 


\begin{tabular}{|c|c|c|c|c|}
\hline$\cdot$ & $\cdot$ & $\cdot$ & $\cdot$ & $\cdot$ \\
\hline$\cdot$ & $\cdot$ & $\cdot$ & $\cdot$ & $\cdot$ \\
\hline$\cdot$ & $\cdot$ & $\cdot$ & $\cdot$ & $\cdot$ \\
\hline$\cdot$ & $\cdot$ & $\cdot$ & $\cdot$ & $\cdot$ \\
\hline$\cdot$ & $\cdot$ & $\cdot$ & $\cdot$ & $\cdot$ \\
\hline
\end{tabular}

(a) Diffusion model

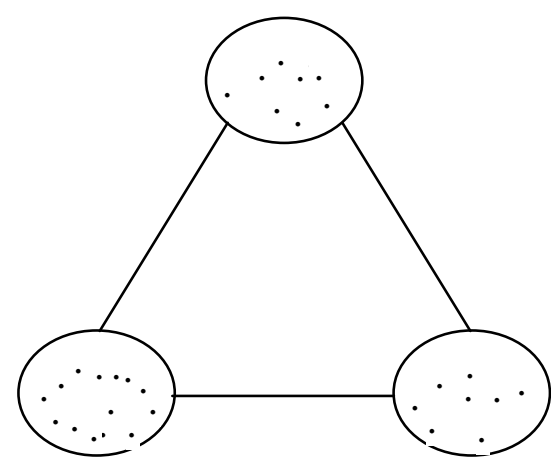

(b) Island model

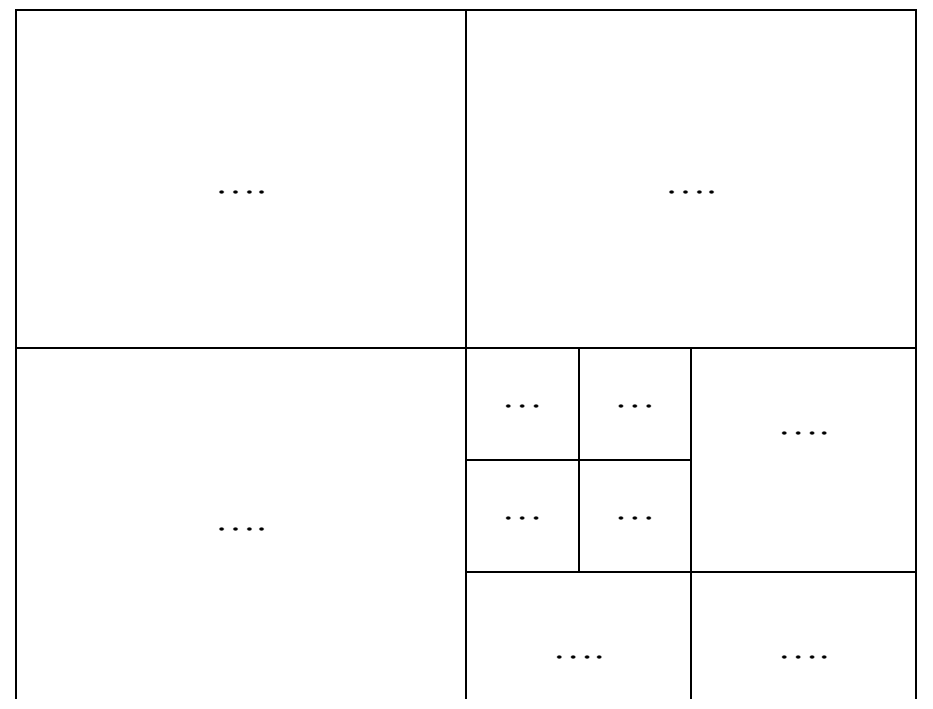

(c) The proposed model

Figure (1): The topologies of structured population in diffusion, island and the proposed models

The diversity of population is reserved by keeping the same number of individuals in each subspace while increasing them in the promising subspaces by dividing them into inner subspaces.

The population size in this model is not based on heuristic strategies. Rather, it is based on discovering inner subspaces in each node of a hypercube where a best or a promising candidate is discovered.

In this model, the number of individuals in each region will depend on the significance of this region and its possibility of having better solutions.

\begin{tabular}{|l|l|l|l|l|l|l|l|l|l|}
\hline $\mathrm{g}_{\mathrm{n}}$ & $\mathrm{g}_{\mathrm{n}-1}$ & $\ldots$. & $\ldots$ & $\ldots$ &.. &.. & $\mathrm{g}_{2}$ & $\mathrm{~g}_{1}$ & $\mathrm{~g}_{0}$ \\
\hline
\end{tabular}

Figure (2): A chromosome structure

Gene number 0

\subsection{Hierarchical Hypercube Structure of the population}

Diffusion model has a one-to-one mapping between individuals and the computational units. On the other hand, the island model has many-to-one mapping between individuals and the computational units as shown in figure (1). Both models have fixed topology that reflects the space structure of the processors or the computational units.
Conversely, the proposed model has a dynamic structure; it starts with the main space that includes all possible chromosomes. The main space, or any other subspace, can be split into a hypercube of adjacent subspaces. The adjacent, or neighbor, subspaces share the property that they have the same parent subspace. Each subspace represents portion of the search space that is determined by genes or parameters values (or genes expression). Based on fitness measurements, a decision can be taken, as will be described in this paper, for 
deepening the exploration in a certain subspace. Any subspace is then divided into a local hypercube of inner subspaces that spans the original subspace; the details of these processes will be described.

The chromosome structure is presented in figure (2) where each subspace can be generally split or divided into ndimensional cube. In order to illustrate the idea, it is assumed that each hypercube is 2-dimensional, or a square. Based on representing the hypercube as a square, this hierarchical structure resembles the Quadtree.

The example of figure (1.c) is represented in figure (3) as hierarchical structure of hypercube in which each node can be divided into an $\mathrm{n}$-dimensional hypercube. The main space has a gene expression in which all genes are represented by the symbol '*'. This symbol means that the corresponding gene can be set to either 0 or 1 . The inner subspaces of the main space can have the genes values of $\{0,1\}$, which implies that each individual in any inner subspace should coincide with its gene expression. In other words, the individual's chromosome in any subspace should match its gene expression at genes equal zero or one, and the gene could be zero or one if the corresponding gene in the genes expression is set to *.

The number of individuals is set to fixed number of subpopulation for every leaf sub-space. The leaf subspace is the space that is not split into a hypercube of inner subspaces. The leaf subspaces represent the active subspaces that are subject to all GA operations. Additionally, each hypercube of inner subspaces can be added or deleted (based on some performance criteria) at any leaf subspace. This process illustrates the dynamic structure of population in our proposed system and the ability to direct the search towards the promising regions in which local optimal solutions are found. At the same time, it could escape from local solutions by keeping the GA search in other subspaces.

Finally, the terminologies that will be used in this approach are summarized as follows:

The main space is the space that includes all the possible chromosomes.

Genes expression: Each space, whether it is the main space or a subspace, has a gene expression that distinguishes it. The genes expression is the list of all genes in the chromosome in which each gene is a member of the set $\{0,1, *\}$. If it is 0 or 1 , this means that all individuals' corresponding gene should be 0 or 1 . If it is *, this symbol means that the individual's gene can be set to either 0 or 1 . Obviously, The main space has a gene expression in which all genes are represented by the symbol '*'.

A subspace is a space in which its gene expression contains zeros and ones. In other words, some genes are restricted to the value of zero or one.

Inner subspace: Each space or subspace can be divided into inner subspaces. The inner space of a space is the space in which all its individuals are also belonging to the upper space. More formally, its genes expression matches the genes expression of the upper space except that some genes symbols "*" in the upper space are replaced by zeros or ones in the inner subspace.

Parent subspace: The parent subspace is the upper space of an inner subspace.

Adjacent subspace: The adjacent, or neighbor, subspaces is the space that shares the property with the subspace that they have the same parent subspace

Leaf subspace: The leaf subspace is the subspace that is not split into a hypercube of inner subspaces.

Internal mating: In internal mating, mating is based on two parents that are picked up from the same subspace

Local mating: In local or adjacent mating, one parent is chosen from the subspace, and the other will be chosen from an adjacent subspace.

\subsection{The proposed Algorithm}

The proposed algorithm starts by initializing the main space with a random population and calculating the fitness of its individuals as shown in the first two steps of the algorithm. Subsequently, the cycle of GA is applied for each leaf subspace (the main space will be considered a leaf subspace at first). In our model, the mating process is divided into internal mating and local mating as described in the following section. After the GA cycle is applied to all leaf subspaces, the hierarchical hypercube of population is maintained based on the condition that a subspace needs to be split for more exploration. In this case, this space will be split. If the number of maximum leaf subspaces is reached, then the worst leaf hypercube (based on another criterion) will be eliminated.

The flow of the program works as follows: The first two steps are executed, which initialize the main space. Then the main loop of the program is executed till a termination condition is reached, which can be maximum number of generations or time out etc. The loop has two parts: The first part is applying all the GA operation over each active or leaf subspace, while the second part maintains the hierarchical structure of the active subspace (whether to split or delete a leaf subspace). The first part applies the GA operations on each subspace: The selection, mating, mutation and evaluation operations. The mating is divided into internal and local mating as will be explained. The flow of the second part works as follows: if any promising individual is found in any leaf space, then this space is split into hypercube of subspaces. But if the maximum number of active leaves is reached, then the worst leaf hypercube is deleted while returning back or activating its parent subspace.

The basic parameters that should be determined for the algorithm are as follows:

Hypercube dimension: $d$

Maximum number of leaf subspaces: MaxL

Number of parent couples in a subspace for internal mating: $n$ 
Main space

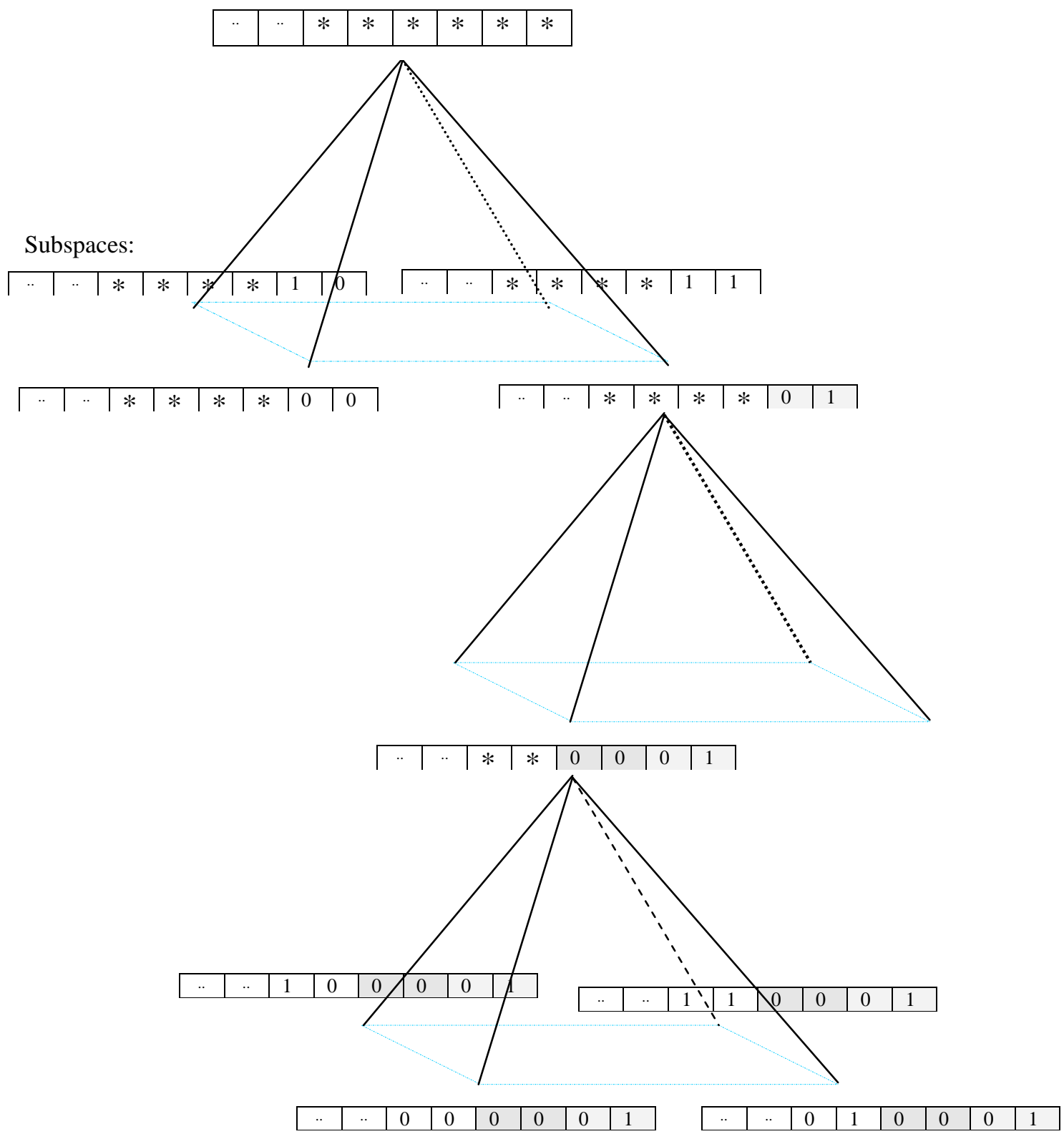

Figure (3): A Hierarchical hypercube structure of the population in the proposed model

Based on the basic parameters, the following parameters can de determined:

- $\quad$ Number of internal parent (for internal mating) $n_{p}$ : $n_{p}=2 n$

- $\quad$ Number of parent (for local mating): $d * n_{p}=2 d n$

- $\quad$ Total number of parent in a subspace: $(d+1) * n_{p}=$ $2(d+1) n$

- $\quad$ Total number of chromosomes in a subspace $n_{s}$ : $n_{s}=2(d+1) n_{p}=4(d+1) n$

- Maximum allowed number of chromosomes: $\operatorname{MaxLn}_{s}=4 \operatorname{MaxL}(d+1) n$

\subsection{The GA operations of the Proposed Model}

\subsubsection{Population Initialization}

The initialization of a subpopulation is not an initial step; rather it occurs whenever a subspace is created while the model is running. Each sub-population of a subspace is generated randomly based on the condition that its individuals should belong to the subspace. An example of individuals generated for the subspace $* * 1 * 0 * 1 * * * 1$ is illustrated in figure (4). 


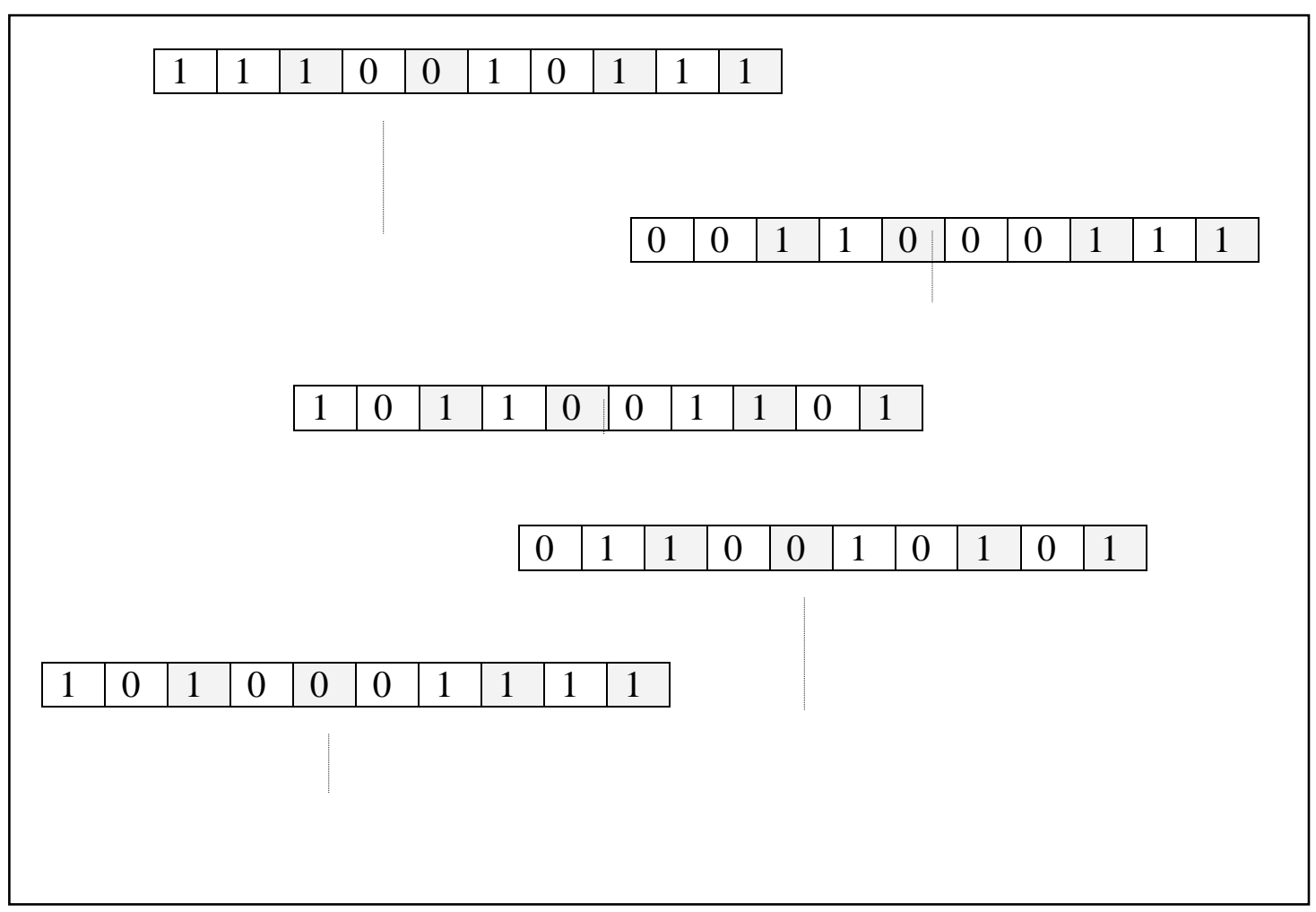

Figure (4): An example of a population that belongs to the subspace $* * 1 * 0 * * 1 * 1$

Subspace S1: **0*1***1*
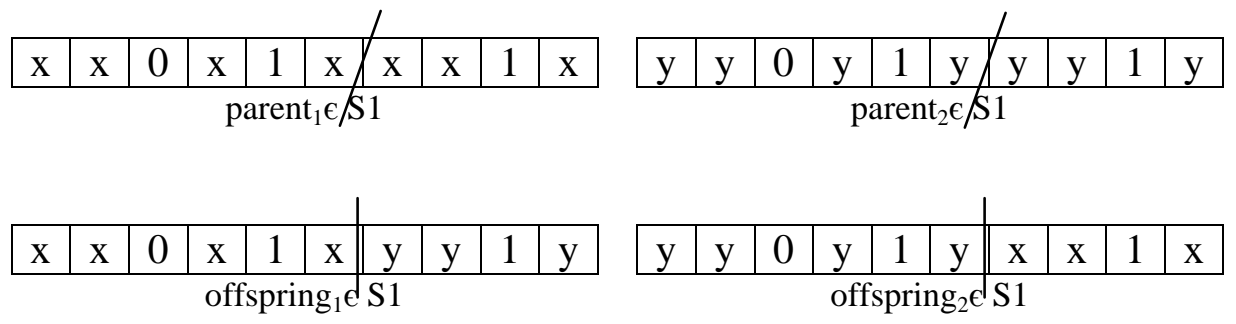

Figure (5): An example showing that internal mating between parents in a subspace yields offspring belonging to the same subspace

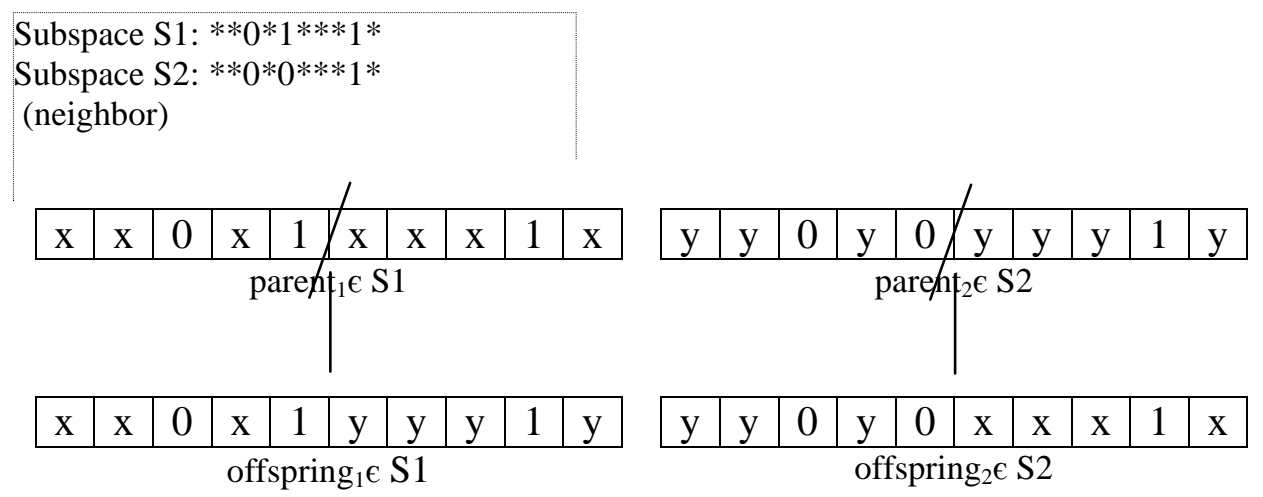

Figure (6): An example showing that local mating between neighbor subspaces yields to two offspring belonging to the same parents' subspaces 


\subsubsection{Selection operation}

The selection process in this model is localized with the ability to interact only with adjacent neighbor subspaces. In other words, for each subspace, individuals can be selected for mating from the same space. Additionally, each subspace should assign individuals to recombine with each adjacent subspace based on their hypercube structure.

\subsubsection{Mating operation}

The model has two types of mating: internal, and local. In internal mating, two parents will be picked up from the same subspace. On the other hand, for local or adjacent mating, one parent is chosen from one subspace, and the other will be chosen from an adjacent subspace.

Since all individuals in a subpopulation share the same genetic information of the genes subspace, then any crossover operation will yield offspring that belong to the same subspace. For example, assume that this subspace has common gene information $* * 1 * 0 * * * 1 *$, as depicted in figure (5), the resulting offspring will be in the same subspace.

On the other hand, for each local mating with any neighbor subspace in this hypercube structure, the resulting two offspring will also belong to these two subspaces. Typically one offspring will belong to a subspace, and the other will belong to the neighbor subspace as illustrated in the example of figure (6):

The proposed hypercube-based population Genetic Algorithm is illustrated as follows:

\section{Module HyperGA}

Initialize_population (subspace MainSpace)

Evaluate_chromosome (subspace MainSpace)

while not terminated ()

for each leaf_subspace $s$

// the GA cycle is applied for

each leaf subspace

Select_parents(subspace $s$ )

Mate_internal(subspace $s$ )

if not isMainSpace $(s)$ then

Mate_local(subspace $s$, neighbors(s))

Mutate(subspace $s$ )

Evaluate_offsprings(subspace $s$ ) end for

if promising_subspace_found(subspace $r$ ) // The dynamic structure of the

hierarchical hypercube is

// maintained here.

// $\mathrm{r}$ represents the subspace that is

needed to be split

leaf_subspace_reached()

if maximum_number_of_

Delete_worst_leaf_hypercube(subspace $t$ )

activate_subspace(subspace $t$ )

end if

Insert_hypercube(subspace $r$ )

end if

end while

end module

\subsubsection{Mutation operation}

In this model, mutation operation is strictly internal. In other words, a restriction is set in order to ensure that the resulted offspring will belong to its subspace. This can be simply done by blocking the dimensions of the subspace from being modified. Figure (7) illustrates this concept, only genes that do not belong to this subspace are allowed to be modified.

\subsubsection{Replacement operation}

The replacement operation in this model is also internal as mentioned in section (3.3.3). Each local mating will yield an offspring in the subspace. On the other hand, each two parents with local mating will produce two offspring. Thus, the population is doubled. Therefore, an ordinary replacement method will be used in order to select half of them. As a result, the new population size will be as the previous population size.

\subsection{A Strategy for subspaces insertion and deletion}

The important questions herein are 'when should the model decide to split a subspace into a hypercube of subspaces?' and 'what is the criteria of choosing the genes dimensions of this hypercube?'.

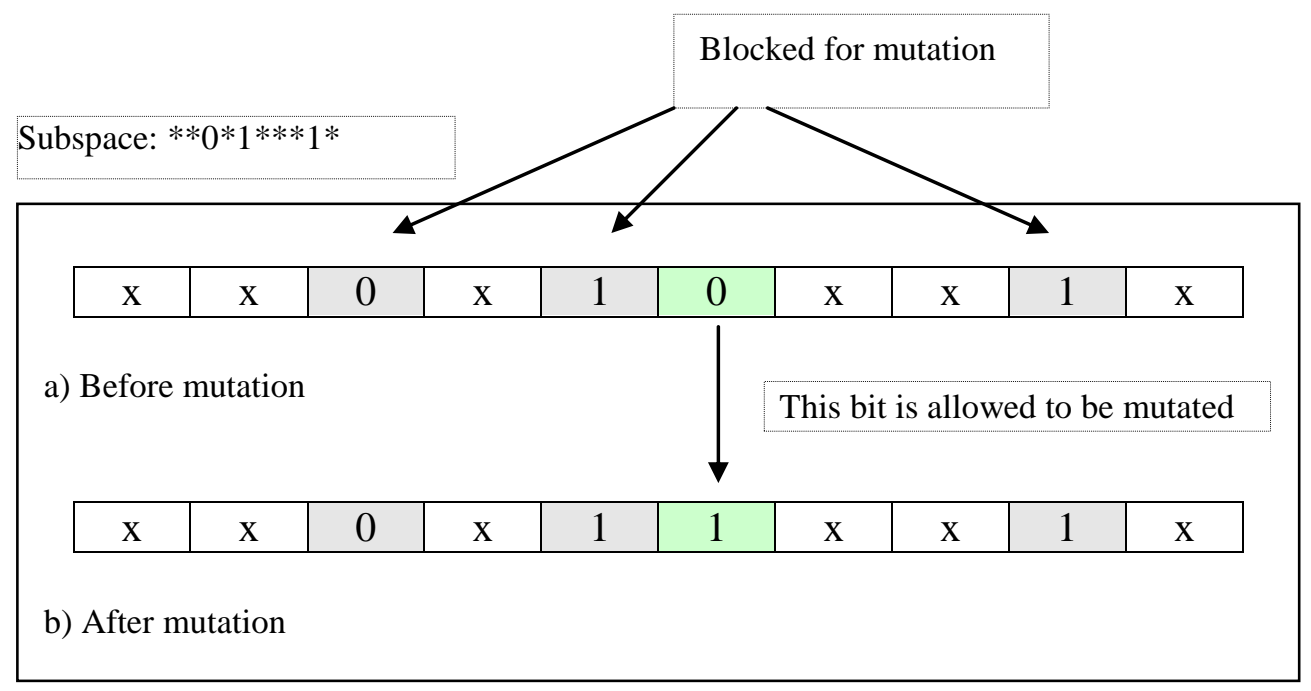

Figure (7): An example showing that the resulting offspring will be in the same subspace after mutation 
In this work, a new hypercube of subspaces is added if a new best fitness is found in a subspace that is better than the current best fitness of the whole population. Consequently, the subspace that contains this new best fitness will be split. This subspace needs more exploration or deepening since a better value is found in it. Specifically, a global variable is used to store the best-found fitness so far. If a subspace produces a better best local-value then this value, then this space will be split into a sub-hypercube.

The genes dimensions that are used for the sub-hypercube creation were randomly chosen. The used strategy for a leaf hypercube of subspaces deletion is to choose the leaf hypercube that has the worst local best fitness for deletion. Then, the best chromosomes of its subspaces are chosen to reconstruct their parent subspace and this subspace will then be activated as a leaf subspace.

\section{SIMULATION AND RESULTS}

In this section, the results of the proposed model will be presented and discussed. The following parameters were chosen as follows:

- The number of chromosomes (the number of chromosomes in a subspace in the proposed model ns): 160

- Hypercube dimension d: 1

- The maximum number of leaf subspaces MaxL: 10

- Mutation probability: 0.01

- $\quad$ Crossover probability: 0.9

- $\quad$ Migration rate (for Island Model): $10 \%$

- The application: 0-1 knapsack problem

- Genes' space: 18 genes.
In this simulation, the following performance metrics were considered and studied: the best fitness, the average fitness and the average deviation (which is measured as the average Hamming distance between the individuals).

The performance of the proposed model was compared with the Standard GA model (SGA), Island GA model (IGA), and the Grid GA model (GGA). The comparison was based on studying the best fitness, average fitness, the average deviation and the population size of each model. The $\mathrm{x}$ axis, or the dependent variable, in this comparison is not the number of generations; rather, it is the number of function evaluations since the population size in the proposed model varies from one generation to the other.

The proposed model was the fastest model to reach the best fitness; the second model was the Grid GA as illustrated in figure (8). On the other hand, the Island Model did not reach this ceiling and the performance of the standard GA was poor.

The average fitness of the four models is shown in figure (9). The average fitness of the proposed model is less than the other models even though it obtained the best fitness faster than the others. Thus, the proposed model balances between reaching the optimal value while preserving the diversity of the population.

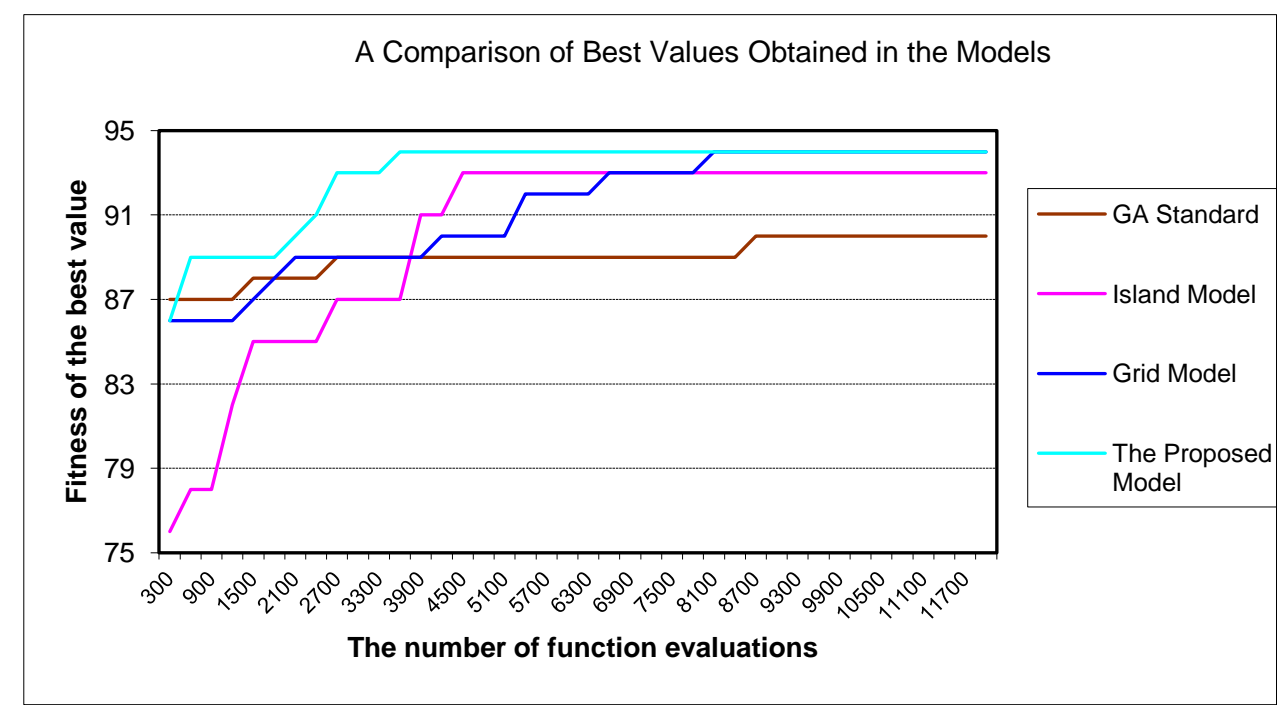

Figure (8): A comparison of the output of the four models in finding the best value based on the number of function evaluations 


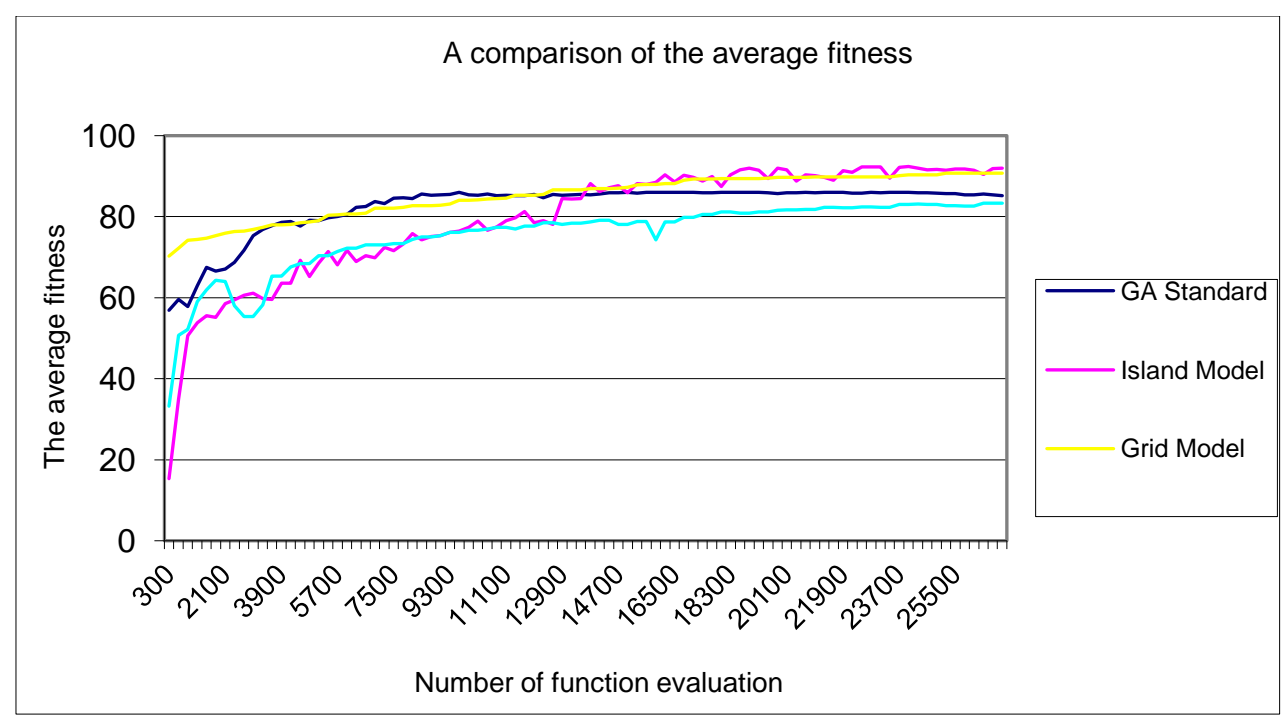

Figure (9): A comparison of the average fitness of the four models based on cost of the number of function evaluations

The comparison between the deviation of chromosomes in each model in figure (10) reveals that the proposed model preserves the diversity whereas the diversity of both island and the standard models tends to reach zero while the grid model has also a good diversity that is comparable to the proposed model.

Figure (11) shows the dynamics or the growth of population size of the proposed model. At the same time, the other models have fixed population size. As mentioned, this increase in population size does not lead to more computational time since the comparison is based on the number of function evaluations.

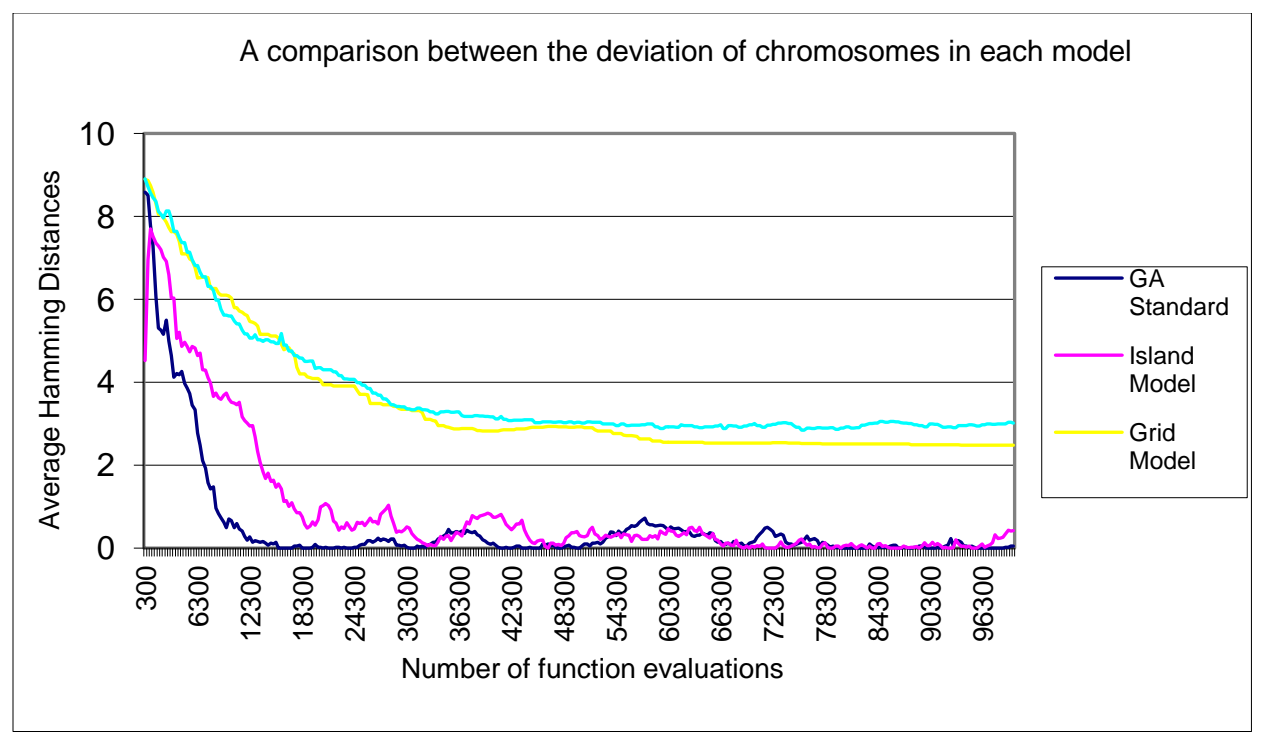

Figure (10): A comparison of the deviation of the four models (based on average mutual hamming distance) versus cost of the number of function evaluations 


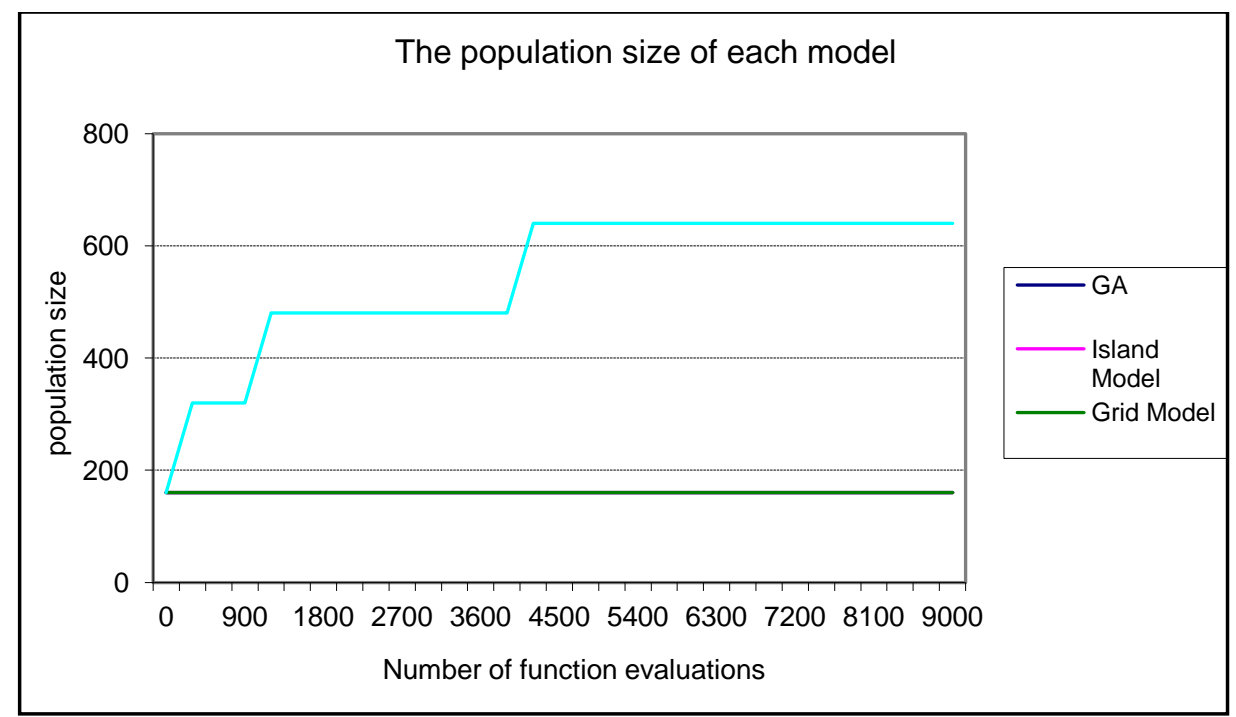

Figure (11): The growth of population size in the proposed model versus other models

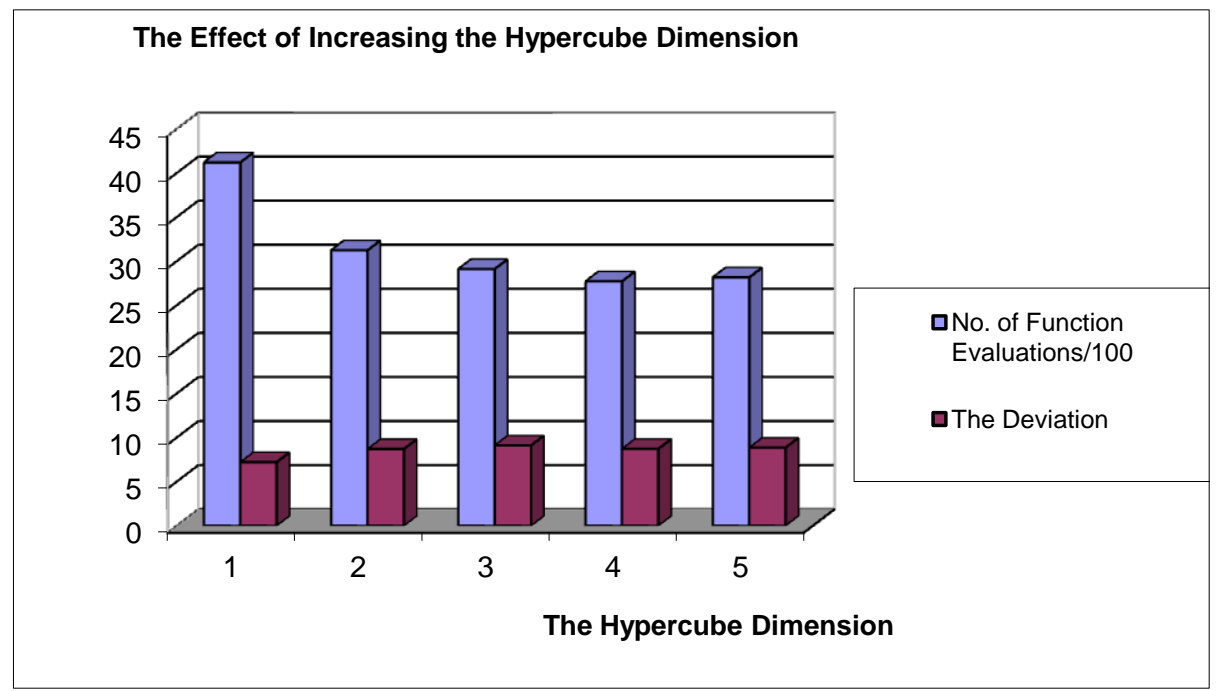

Figure (12): The Effect of Increasing the Hypercube Dimension on the Performance of the Model 


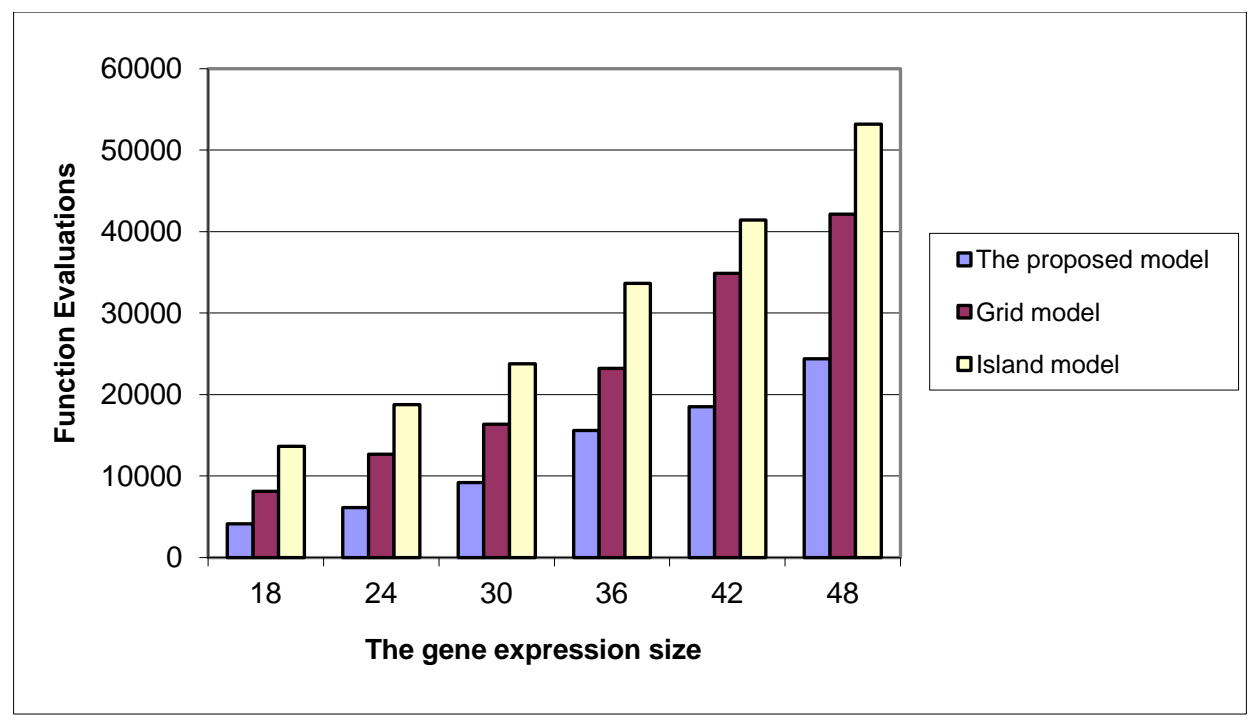

Figure (13): The comparison of the performance with respect to the gene expression size

Table (1): The statistical data of the frequency of each model to reach the optimal value based on 100 different runs.

\begin{tabular}{|c|c|c|c|c|c|c|c|c|c|}
\hline $\begin{array}{c}\text { \# of function } \\
\text { evaluation >> }\end{array}$ & 1000 & 2000 & 4000 & 8000 & 16000 & 32000 & 64000 & $>64000$ & The sum \\
\hline Hyper GA & 5 & 24 & 37 & 26 & 8 & & & & 100 \\
\hline Grid GA & & 4 & 23 & 34 & 27 & 10 & 2 & & 100 \\
\hline Island GA & & & 10 & 18 & 30 & 27 & 15 & & 100 \\
\hline Standard GA & & & 13 & 17 & 16 & 13 & 15 & 26 & 100 \\
\hline
\end{tabular}


Figures (8), (9) and (10) illustrate the balancing of the proposed model between exploration and exploitation. Exploration presents the ability of the model to explore new subspaces, which can be measured by keeping the diversity of the population as shown in figure (10). At the same time, exploitation presents the ability to deepen the search towards the optimal solutions, which can be estimated from the ability to reach an optimal solution quickly as shown in figure (8).

The effect of changing the hypercube dimension is studied. The performance of the model when the hypercube dimension is changing from one to five is presented in figure (12). The performance is represented by the number of function evaluations and the deviation of the population. As shown in figure (12), the best performance is at hypercube dimension equals four.

In figure (13), the performance of the models in case of changing the gene expression size is compared. The proposed model has better performance when the gene expression size is increased.

In order to verify the superiority of the proposed model, 100 runs were conducted on each model. The performance metric is the number of function evaluations needed to reach the best-found fitness. The statistical results are shown in table (1).

Finally, the model was tested with a continuous function. The Rosenbrock's function (f2 testing function) was as a test pad for continuous functions. The 2 function is as follows:

$$
f_{2}\left(x_{1}, x_{2}\right)=100\left(x_{2}-x_{1}^{2}\right)^{2}+\left(1-x_{1}\right)^{2}
$$

Where $-1.5 \leq x_{i} \leq 1.5$. The $\mathrm{f} 2$ function has an optimal value at $(1,1)$.

In this test, dynamic chromosome structure is used. First the chromosome starts with 2 bits. Each bit represents either the positive or the negative part of the variable. Then, after dividing the subspaces, each subspace will add more bits which deepen the search and divides the whole region into more specified ranges depending on the hypercube dimension.

The deviation of the proposed model, when optimizing the f2 function, compared to other models is shown in figure (14). Figure (15) presented the performance of the models in terms of the number of function evaluations.

\subsubsection{Possibilities for future works}

These elementary results showed that the hypercube-based population could lead to better performance. However, more work is needed in order to enhance the performance of the model such as finding and applying:

- An optimal strategy for the insertion/deletion of the internal hypercube.

- An automatic strategy for determining the dimension of the gene space especially for the continuous functions

- An approach for selection the dimension of the hypercube

- Other well-known applications in order to apply and test the model.

On the other hand, this approach could help in automating the process of:

- Controlling the population size
- $\quad$ Directing the search towards the gene spaces where the optimal results may reside in.

- Preventing the search from immature convergence

- Dynamically redistributing the population in regions that could be promising

- Controlling the mating and the mutation processes

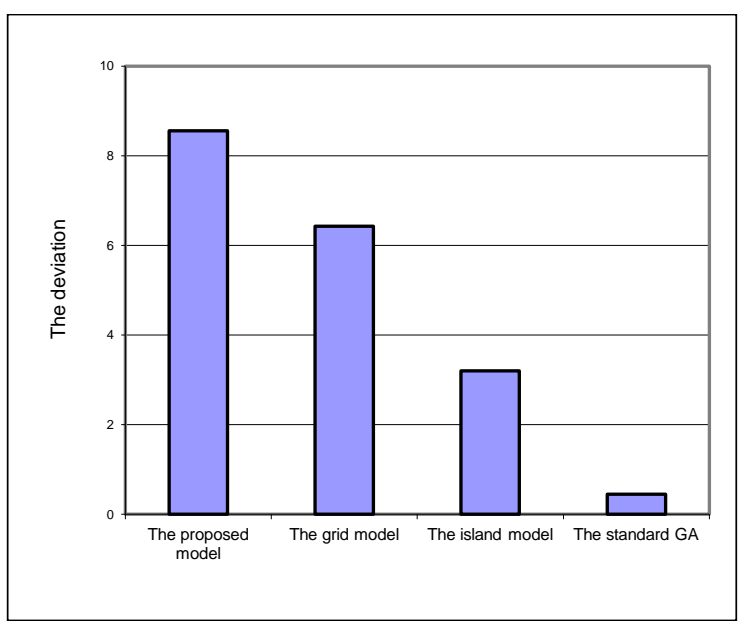

Figure 14: The deviation of the population when optimizing the $\mathbf{2} 2$ function

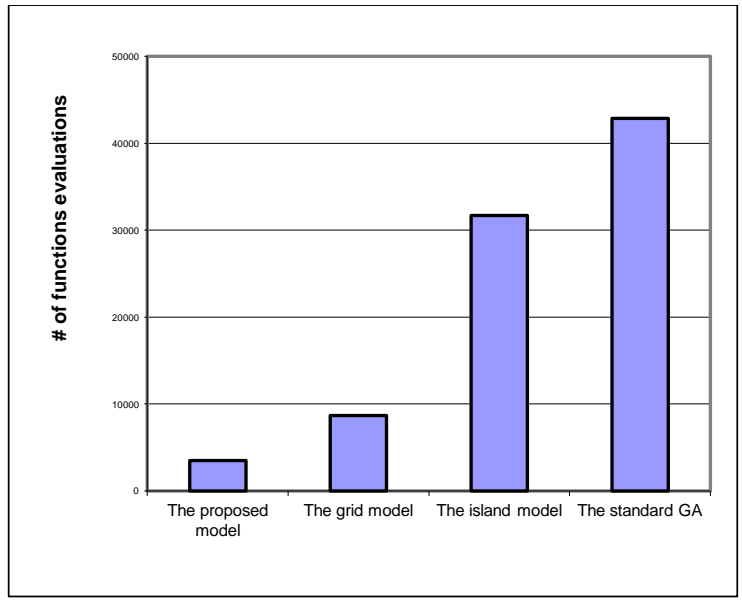

Figure 15: The number of function evaluations when optimizing the $\mathbf{2}$ function

\section{CONCLUSION}

In this paper, a structured population GA approach is introduced. It represents the population as a hierarchy of hypercubes that can dynamically construct its structure. The subpopulation represents a subdivision of the search space or the genes space. This approach could help in controlling the search directions and managing the concentration of individuals over the real search space.

The proposed model was simulated and compared with SGA, IGA and GGA. The findings showed that the proposed model is faster in finding the optimal value. In the mean time, it could preserve the diversity of the individuals and keep a relatively good average fitness value. Finally, a future work is needed in order to find a better strategy for the deletion and the insertion of hypercubes as well as selecting the dimension of the hypercube. 


\section{REFERENCES}

[1] Whitley, D. "Cellular genetic algorithms". In Proceedings of the Fifth International Conference on Genetic Algorithms S. Forrest (Ed.), San Mateo, CA : Morgan Kaufman Publishers,, 1993, pp. 658.

[2] Back, T., Fogel, D. B., Michalewicz, Z., Et Al., Eds. Handbook on "Evolutionary Computation". IOP Publishing Ltd and Oxford University Press, 1997.

[3] Krink, T., Mayoh, B. H., and Michalewicz, Z. "A Patchwork model for evolutionary algorithms with structured and variable size populations". In Proceedings of the Genetic and Evolutionary Computation Conference, Vol. 2, 1999, pp. 1321-1328.

[4] Krink, T., and Ursem, R. K. "Parameter control using the agent based patchwork model". In Proceedings of the Second Congress on Evolutionary Computation (CEC2000). San Diego, CA, USA: 77-83, 2000.

[5] Gorden, V. S., Pirie, R., Wachter, A., and Sharp, S. "Terrain-based genetic algorithm (TBGA): Modeling parameter space as terrain". In Proceedings of the Genetic and Evolutionary Computation Conference, vol. 1, 1999, pp. 299- 235.

[6] René Thomsen, Peter Rickers, Thiemo Krink, "A Religion-Based Spatial Model for Evolutionary Algorithms", proceedings of the 6th International Conference on Parallel Problem Solving from Nature (PPSN VI)

[7] Nagham Azmi AL-Madi, Ahamad Tajudin Khader, "De Jong's Sphere Model Test for A Social-Based Genetic Algorithm (SBGA)", IJCSNS International Journal of Computer Science and Network Security, Vol. 8 No. 3 pp. 179-185, 2008.

[8] Gordon V. S., Whitley D.: "Serial and Parallel Genetic Algorithms as Function Optimizers". In Forrest S. (ed.): Proceedings of the Fifth International Conference on Genetic Algorithms. Morgan Kaufmann, San Mateo, CA (1993) 177-183

[9] Enrique Alba, Antonio J. Nebro, and Jose' M. Troya, "Heterogeneous Computing and Parallel Genetic Algorithms", Journal of Parallel and Distributed Computing 62, 1362-1385 (2002)

[10] Paulo M. Franca, Jatinder N.D. Gupta, Alexandre S. Mendes, Pablo Moscato, Klaas J. Veltink, "Evolutionary algorithms for scheduling a flowshop manufacturing cell with sequence dependent family setups", Computers \& Industrial Engineering 48, Elsevier (2005) 491-506

[11] Daniel Ashlock, Mark Smucker, and John Walker, Graph Based Genetic Algorithms, in Proceedings of the Congress on Evolutionary Computation: (1999) 13621368.

[12] Manderik, B. and Spiessens, P., "Fine-Grained Parallel Genetic Algorithms", Proceedings of the 3rd International Conference on Genetic Algorithms, 1989.

[13] Hillis D., "Co-Evolving Parasites Improve Simulated Evolution as an Optimization Procddure", physics D 42 (1990): 228-234.

[14] Collins R. and Jefferson, D., "Selection in Massively Parallel Genetic Algorithms", Proceedings of the 4th
International Conference on Genetic Algorithms, Morgan-Kaufmann (1991), pp. 249-256.

[15] E. K. Burke, S. Gustafson, and G. Kendall. Diversity in genetic programming: An analysis of measures and correlation with fitness. IEEE Transactions on Evolutionary Computation, 8(1):47-62, 2004.

[16] E. Zitzler, K. Deb, and L. Thiele. Comparison of multiobjective evolutionary algorithms: Empirical results. Evolutionary Computation, 8(2):173-195, 2000

[17] Y. Leung, Y. Gao, and Z. B. Xu. Degree of population diversity - a perspective on premature convergence in genetic algorithms and its Markov chain analysis. IEEE Transactions on Neural Networks, 8(5):1165-1176, 1997.

[18] J. C. Costa, R. Tavares, and A. Rosa. An experimental study on dynamic random variation of population size. In IEEE International Conference on Systems, Man, and Cybernetics, pages 607-612, 1999.

[19] A. Piszcz and T. Soule. Genetic programming: Optimal population sizes for varying complexity problems. In Generic and Evolutionary Computation Conference, pages 953-954, 2006.

[20] V. K. Koumousis and C. P. Katsaras. A saw-tooth genetic algorithm combining the effects of variable population size and reinitialization to enhance performance. IEEE Transactions on Evolutionary Computation, 10(1):19-28, 2006

[21] F. G. Lobo and C. F. Lima. A review of adaptive population sizing schemes in genetic algorithms. In Genetic and Evolutionary Computation Conference, pages 228-234, 2005.

[22] G. R. Harik and F. G. Lobo. A parameter-less genetic algorithm. In Genetic and Evolutionary Computation Conference, pages 258-265, 1999.

[23] B. Thomas, A. Eiben, and V. der Vaart. An empirical study on GAs without parameters. In Parallel Problem Solving from Nature V, pages 315-324, 2000.

[24] Espinoza, F., Minsker, B. S. and Goldberg, D. A SelfAdaptive Hybrid Genetic Algorithm. Proceedings of the Genetic and Evolutionary Computation Conference, San Francisco, Morgan Kaufmann Publishers, p. 759, (2001).

[25] Krink, T. and Ursem, R. K. Parameter Control Using the Agent Based Patchwork Model. Proceedings of the Congress on Evolutionary Computation, pp. 77-83, (2000).

[26] Kee, E., Airey, S. and Cye, W. An Adaptive Genetic Algorithm. Proceedings of the Genetic and Evolutionary Computation Conference, pp. 391-397, (2001).

[27] Tanese, R. Distributed Genetic Algorithms. In Proceedings of the Third International Conference on Genetic Algorithms, pp. 434-439, (1989).

[28] H. Homayounfar, S. Areibi, F. Wang, "An Advanced Island Based GA For Optimization Problems", DCDIS Conference Guelph, Ontario, Canada, pp: 46-51, May 2003.

[29] Shisanu Tongchim, Prabhas Chongstitvatana: Parallel genetic algorithm with parameter adaptation. Inf Process. Lett. 82(1): 47-54 (2002) 
[30] Dirk Schlierkamp-Voosen and Heinz Mühlenbein (1994). Strategy adaptation by competing subpopulations. PPSN 3, pages 199-208.

[31] Cantu'-Paz, E., Designing efficient and accurate parallel genetic algorithms, $\mathrm{PhD}$ thesis, Graduate College of the University of Illinois at Urbana Champaign 1999.

[32] Malott B., Averill R.C., Goodman E.D., Ding Y., Punch W.F., Use of Genetic Algorithms for optimal design of laminated composite sandwich panels with bendingtwisting coupling, presented at AIAA SDM (Structures, Dynamics and Materials), Apr 96, 1996

[33] Eby D., Averill R., Goodman E., and Punch W., The Optimization of Flywheels Using an Injection Island Genetic Algorithm, in Bentley, P. (ed.), Evolutionary Design by Computers, Morgan Kaufmann, San Francisco, 1999, pp.167-190

[34] Z. Skolicki and K. D. Jong. Improving evolutionary algorithms with multi-representation island models. In Parallel Problem Solving from Nature - PPSN VIII 8th International Conference. Springer-Verlag, 2004.

[35] D. Whitley, S. Rana, and R. B. Heckendorn. Island model genetic algorithms and linearly separable problems. In Selected Papers from AISB Workshop on Evolutionary Computing, volume 1305 of Lecture Notes In Computer Science, pages 109- 125. Springer-Verlag, 1997.

[36] Cengiz Kahraman, Orhan Engin, Ihsan Kaya, Mustafa Kerim Yilmaz. An application of effective genetic algorithms for Solving Hybrid Flow Shop Scheduling Problems. International Journal of Computational Intelligence Systems, 1(2): 134-147, 2008.

[37] Alireza Fasih, Jean Chamberlain Chedjou, Kyandoghere Kyamakya. Cellular Neural Networks-Based Genetic Algorithm for Optimizing the Behavior of an Unstructured Robot. International Journal of Computational Intelligence Systems, 2(2): 124-131, 2009
[38] Yanbing Liu, Jun Huang. A Novel Fast Multi -objective Evolutionary Algorithm for QoS Multicast Routing in MANET. International Journal of Computational Intelligence Systems, 2(3): 288-297, 2009.

[39] Nikolas Geroliminis, Konstantinos Kepaptsoglou, Matthew G. Karlaftis, A hybrid hypercube - Genetic algorithm approach for deploying many emergency response mobile units in an urban network, Elsevier, pp 278-300, doi:10.1016/j.ejor.2010.08.031, 2010.

[40] ystein Langangen, Eric Edeline, Jan Ohlberger, Ian J. Winfield, Janice M. Fletcher, J. Ben James, Nils Chr. Stenseth, L. Asbjorn Vollestad, Six decades of pike and perch population dynamics in Windermere, Elsevier, pp 131-139, doi:10.1016/j.fishres.2011.01.029, 2011

[41] Michael Barfield, Robert D. Holt and Richard Gomulkiewicz, Evolution in Stage-Structured Populations, The American Naturalist, vol. 177, no. 4, pp. 397-409, DOI: 10.1086/65890, April 2011

[42] Ting Y. Lim, Structured population genetic algorithms: a literature survey, Artificial Intelligence Review pp. 115, doi:10.1007/s10462-012-9314-6, February 2012

[43] XianBin CAO, YuanPing GUO and Hong QIAO, A New Strategy of Dynamically Adjusting Population Size for Coevolutionary Algorithms, InternationalJournal of Intelligent Control and Systems, vol. 10, no. 3, pp 251 257, September 2005

[44] Isabel Gordo and Paulo R. A. Campos, Adaptive evolution in a spatially structured asexual population, Springer, Genetica (2006) 127:217-229, DOI 10.1007/s10709-005-4012-9, 2006

[45] F.G. Lobo and C.F. Lima, Adaptive Population Sizing Schemes in Genetic Algorithms, Studies in Computational Intelligence (SCI) 54, 185-204, SpringerVerlag Berlin Heidelberg, 2007 